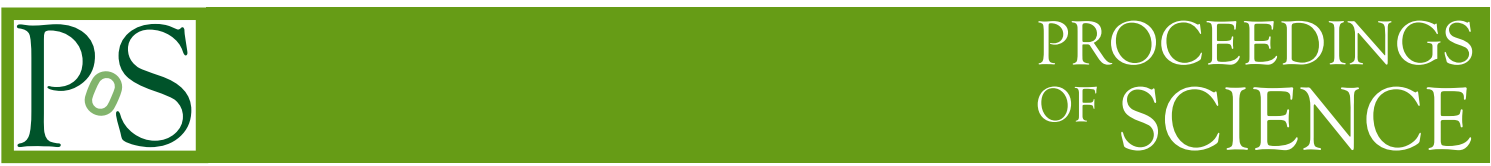

\title{
Project P2 - The weak charge of the proton
}

\section{Becker*}

Institute for Nuclear Physics, University of Mainz

E-mail: beckerd@kph.uni-mainz.de

\section{K. Gerz, S. Baunack, K. S. Kumar, F. E. Maas}

\begin{abstract}
The goal of Project P2 is to determine the electroweak mixing angle $\sin ^{2}\left(\theta_{W}\right)$ to a world beating precision of $0.15 \%$ at low momentum transfer $\left(Q^{2}=0.003 \mathrm{GeV}^{2}\right)$, utilizing the upcoming MESA accelerator facility in Mainz. The experimental method comprises a measurement of the proton's weak charge $Q_{W}^{p}$ to a relative uncertainty of $1.9 \%$ via the parity violating asymmetry in elastic electron-proton scattering. We are going to present the experimental method and discuss the achievable precision within Project P2. Furthermore, we will show results of Geant4 simulations which were carried out to study a possible experimental setup.
\end{abstract}

52. International Winter Meeting on Nuclear Physics - Bormio 2014,

27-31 January 2014

Bormio, Italy

${ }^{*}$ Speaker. 


\section{Introduction}

The electroweak mixing angle $\sin ^{2}\left(\theta_{W}\right)$ may be regarded as the most important parameter in the theory of electroweak interactions. In order to test the Standard Model's prediction and to determine the value of the electroweak mixing angle, numerous experiments have been performed over a wide range of energies. Figure 1 gives an overview of recent measurements of $\sin ^{2}\left(\theta_{W}\right)$ as well as future projects.

Next generation projects like for example the Møller Experiment and P2 are going to determine the electroweak mixing angle to a precison high enough to compare the results to predictions of models going beyond the Standard Model. It is pointed out in [2] that high precision measurements of the electron's and proton's weak charges provide complementary access to new physics. Figure 2 shows the shifts of the electron's and proton's weak charges predicted by new physics models.

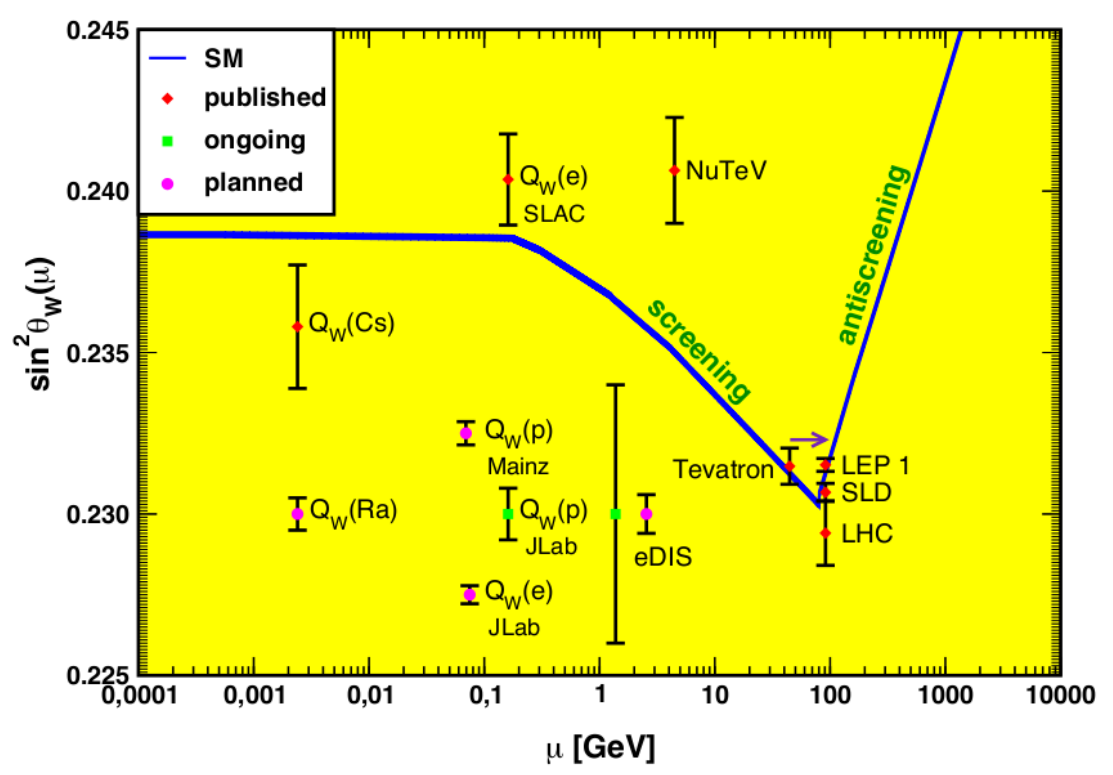

Figure 1: An overview of recent determinations and future measurements of the electroweak mixing angle (courtsey of Jens Erler): The absorption of universal quantum corrections into $\sin ^{2}\left(\theta_{W}\right)$ leads to a running of the electroweak mixing angle w.r.t. the energy scale $\mu$. The vertical placement of the planned/ongoing projects' points in this picture is arbitrary, their error bars depict the projected uncertainties. The Møller Experiment [1] $\left(\mathrm{Q}_{W}(\mathrm{e}), \mathrm{Jlab}\right)$ aims to measure $\sin ^{2}\left(\theta_{W}\right)$ via the electron's weak charge to a relative uncertainty of $0.1 \%$. The goal of Project $\mathrm{P} 2\left(\mathrm{Q}_{W}(\mathrm{p})\right.$, Mainz) is to measure the weak charge of the proton with a precision of $1.9 \%$ which corresponds to a precision of $0.15 \%$ in the determination of the electroweak mixing angle. The two measurements are complementary in terms of searching for physics beyond the Standard Model.

\section{Experimental method and achievable precision}

The P2 Experiment is going to measure the weak charge of the proton through the parity violating asymmetry $A^{P V}$ in elastic electron-proton scattering. Asymmetry measurements are a well established experimental technique. Figure 3 illustrates the basic concept. 

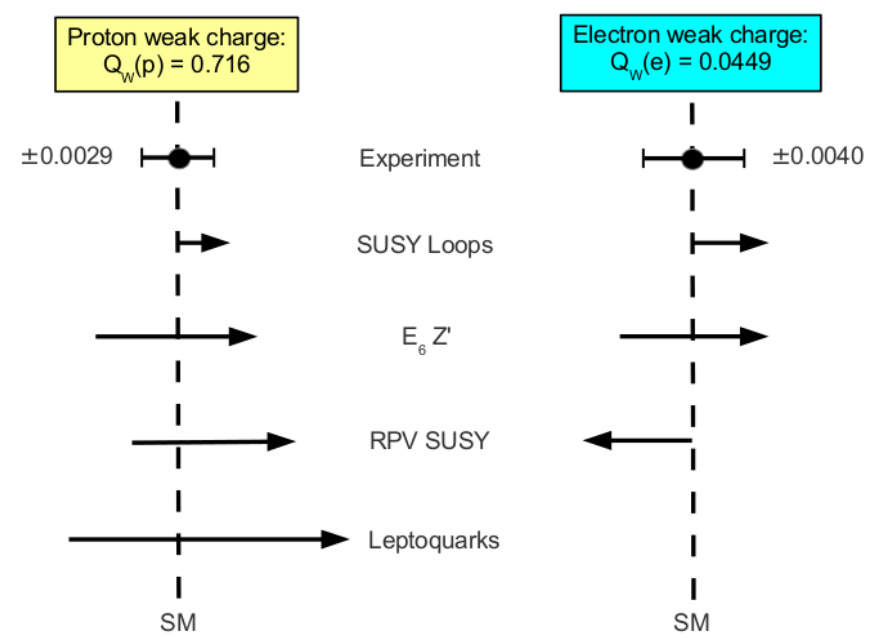

Figure 2: Shifts of the proton and electron weak charges predicted by new physics models according to [2]: The dashed lines mark the Standard Model values of the weak charges. The arrows depict possible deviations from the Standard Model values. Since the shifts differ in size and direction for the models and the weak charges, high precision measurements of their values will allow for a test of these predictions.

The parity violating asymmetry depends on the proton's weak charge $Q_{W}^{p}$ in the following manner:

$$
A^{P V}=\frac{-G_{F} Q^{2}}{4 \pi \sqrt{2} \alpha}\left(Q_{W}^{p}-F\left(Q^{2}\right)\right)
$$

with

$$
F\left(Q^{2}\right)=F_{\text {em }}\left(Q^{2}\right)+F_{\text {axial }}\left(Q^{2}\right)+F_{\text {strange }}\left(Q^{2}\right) .
$$

$F\left(Q^{2}\right)$ is the sum of hadronic contributions stemming from the nucleon's inner structure. $G_{F}$ is the Fermi-coupling and $Q^{2}$ is the negative squared 4-momentum transfer. At low values of $Q^{2}$

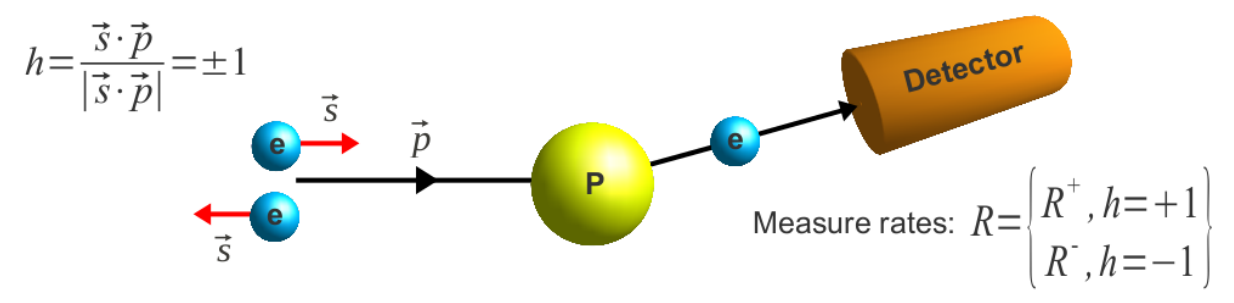

Figure 3: Experimental method of Project P2: Beam electrons with helicity +1 or -1 are scattered off unpolarized protons and then registered in a counting detector, so that one observes rates $R^{+}$or $R^{-}$. The main contributions to the scattering amplitude are stemming from the electromagnetic and the weak interaction. Since the weak interaction violates parity, the cross sections $\sigma^{+}$and $\sigma^{-}$and therefore the rates $R^{+}$and $R^{-}$ are not the same. The parity violating asymmetry in elastic electron-proton scattering may be defined as $A^{P V}=\frac{\sigma^{+}-\sigma^{-}}{\sigma^{+}+\sigma^{-}}$and is in the order of $10^{-8}$ for P2's projected experimental conditions. 


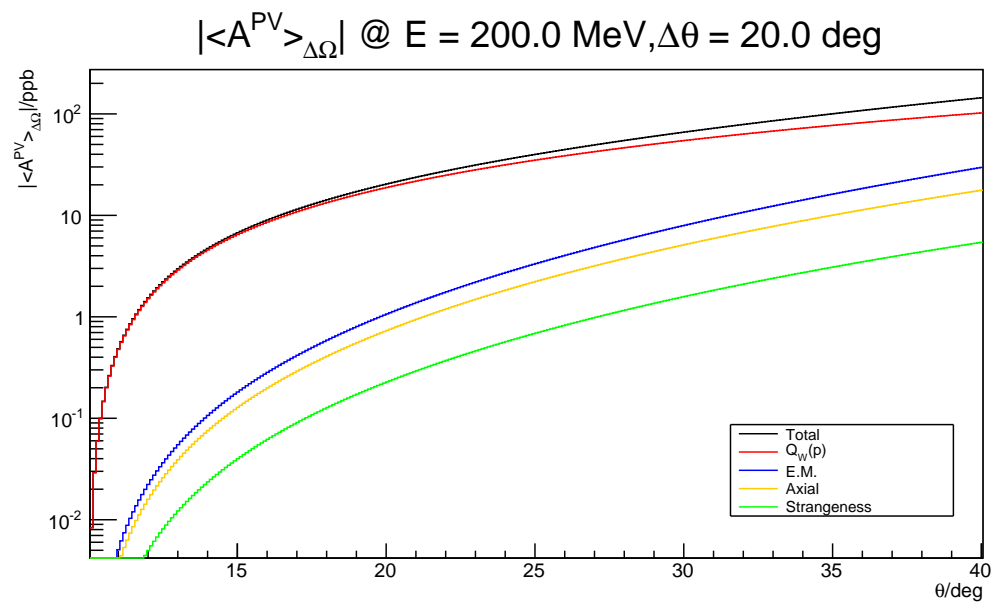

Figure 4: Parity violating asymmetry in elastic electron-proton scattering, averaged over solid angle for a beam energy of $200 \mathrm{MeV}$ and a detector acceptance of $\pm 10^{\circ}$, vs. central scattering angle $\theta$. The total asymmetry is shown in black, the contribution of the proton weak charge in red. Nucleon structure contributions are shown as well. At low central angles around $20^{\circ}$ the asymmetry is clearly dominated by the contribution stemming from the proton's weak charge.

the hadronic contributions become very small, see figure 4 .

So a measurement of $A^{P V}$ at low momentum transfer grants access the the proton's weak charge, which is (at tree level) given by

$$
Q_{W}^{p}=1-4 \cdot \sin ^{2}\left(\theta_{W}\right),
$$

and therefore to the electroweak mixing angle.

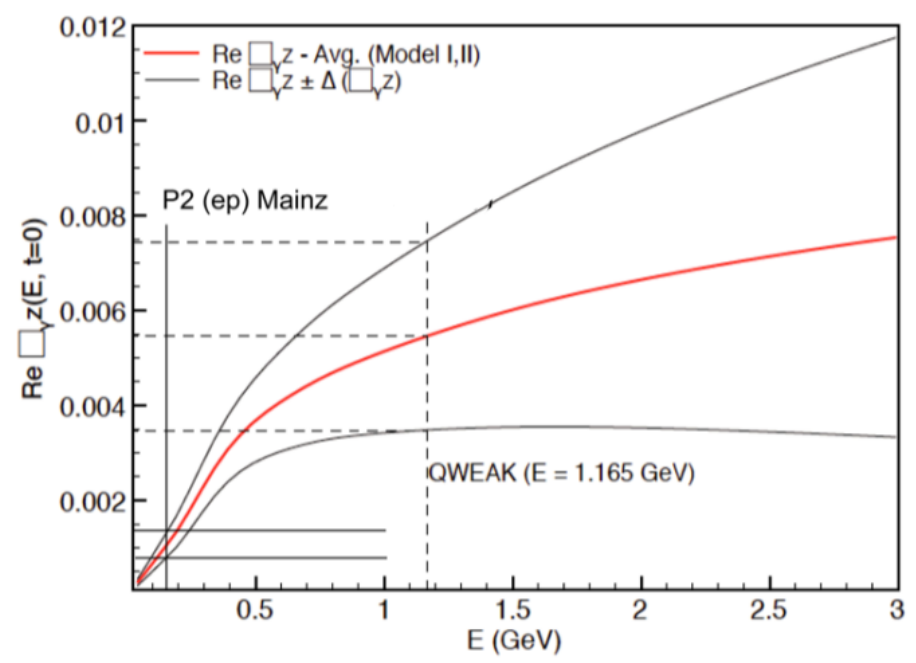

Figure 5: $\gamma$-Z-boxgraph correction to the weak charge of the proton, taken from [3]. Shown is the correction (middle curve, red) and the uncertainty bands. With increasing beam energy, both mean value and uncertainty of the contribution rapidly grow, while the mean value is small and the uncertainty is well under control at low beam energies. 
In order to achieve a low value for $\mathrm{Q}^{2}$, one generally has two options: Perform the measurement at high beam energy choosing a small scattering angle or choose a small beam energy which allows the scattering angle to be larger. The $\mathrm{P} 2$ collaboration decided to perform the measurement at low beam energies around $200 \mathrm{MeV}$, because here the uncertainty of the $\gamma$-Z-boxgraph contribution to the proton weak charge is very small. Figure 5 shows a calculation of the energy dependence of the correction.

To predict the achievable precision in the determination of $\sin ^{2}\left(\theta_{W}\right)$, the $\mathrm{P} 2$ collaboration has performed error propagation calculations using Monte Carlo methods. The calculations are based on the following ansatz, which is commonly used in parity violation experiments:

$$
A^{\text {exp }}=P \cdot\left\langle A^{P V}\right\rangle_{L, \Delta \Omega}+A^{a p p}
$$

$A^{\exp }$ is the experimentally observed asymmetry, $P$ the polarization of the electron beam, $\left\langle A^{P V}\right\rangle_{L, \Delta \Omega}$ the parity violating asymmetry averaged over solid angle $\Delta \Omega$ and target length $L, A^{a p p}$ the sum of accelerator induced so called false asymmetries stemming mainly from helicity correlated fluctuations of the electron beam's properties. Solving equation 2.4 for $\sin ^{2}\left(\theta_{W}\right)$ leads to an expression of the form:

$$
\sin ^{2}\left(\theta_{W}\right)=\zeta\left(A^{e x p}, A^{a p p}, P, E_{\text {beam }}, \Delta \Omega, L, F_{\text {em }}, F_{\text {axial }}, F_{\text {strange }}\right),
$$

where $\zeta$ is a map of the parameters described in the text above. One may now assign a random distributions to each of $\zeta$ 's parameters and vary them accordingly in equation 2.5. For a single variation of all of $\zeta$ 's parameters, one obtains the following expression:

$$
\begin{aligned}
\sin ^{2}\left(\theta_{W}\right)+\delta \sin ^{2}\left(\theta_{W}\right)= & \zeta\left(A^{\text {exp }}+\delta A^{\text {exp }}, A^{a p p}+\delta A^{a p p}, P+\delta P\right. \\
& E_{\text {beam }}+\delta E_{\text {beam }}, \Delta \Omega+\delta \Delta \Omega, L+\delta L \\
& \left.F_{\text {em }}+\delta F_{\text {em }}, F_{\text {axial }}+\delta F_{\text {axial }}, F_{\text {strange }}+\delta F_{\text {strange }}\right),
\end{aligned}
$$

where $\delta \sin ^{2}\left(\theta_{W}\right)$ has to be chosen so that the equation holds. Iterating this procedure while storing the values $\delta \sin ^{2}\left(\theta_{W}\right)$ leads to a distribution of $\sin ^{2}\left(\theta_{W}\right)$ at the point in the parameter space that corresponds to the chosen expectation values of $\zeta$ 's parameters. From this distribution the standard deviation $\Delta \sin ^{2}\left(\theta_{W}\right)$ may be extracted. In this manner, the simultaneous variation of all parameters leads to the total uncertainty in the electroweak mixing angle at a certain point in the parameter space. The variation of a single parameter with all other parameters fixed at their expectation values will give the contribution of that parameter to the total uncertainty. Figure 6 shows the result of a systematic change in the expectation value of the central scattering angel with fixed expectation values for the beam energy and the width of the detector acceptance. The calculations have been done for the proposed experimental conditions of Project P2, which are summarized in table 1 . The minimum value of the realtive uncertainty of $\sin ^{2}\left(\theta_{W}\right)$ in figure 6 is

$$
\frac{\Delta \sin ^{2}\left(\theta_{W}\right)}{\sin ^{2}\left(\theta_{W}\right)}=0.15 \%
$$




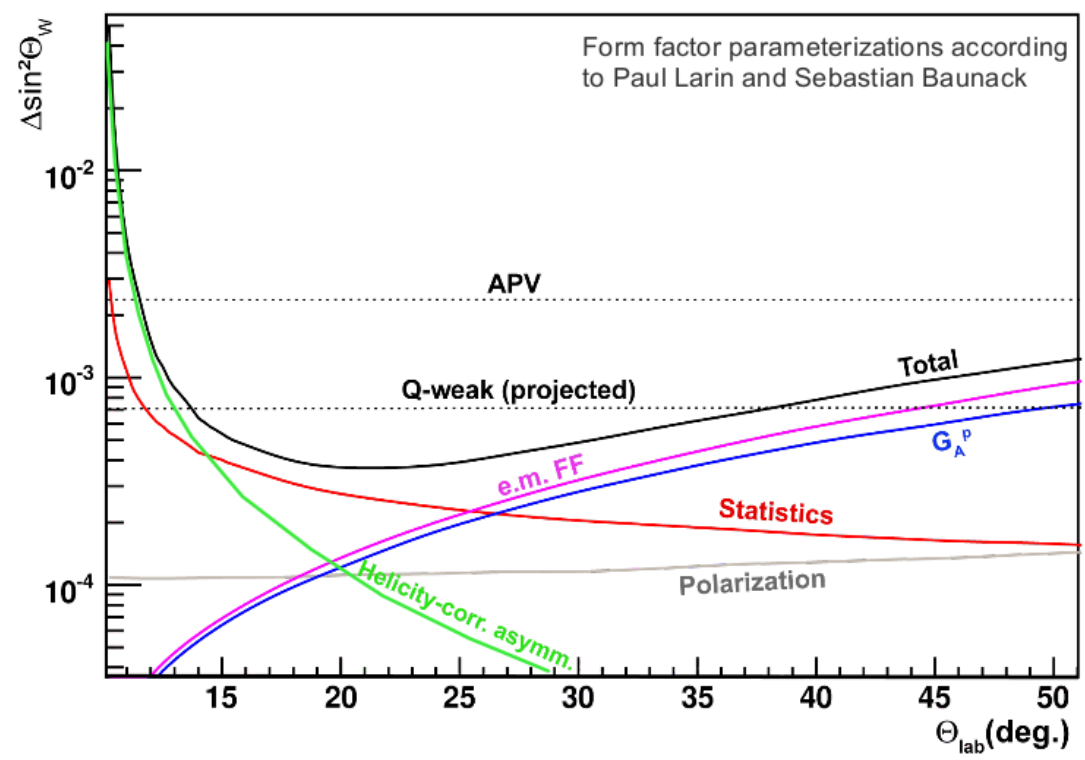

Figure 6: Results of the error propagation calculations for the proposed experimental conditions (see table 1) of P2: Shown is the uncertainty $\Delta \sin ^{2}\left(\theta_{W}\right)$ of the electroweak mixing angle (Total) vs. the central scattering angle in the laboratory frame for a fixed beam energy of $200 \mathrm{MeV}$. For each point on the $\Theta_{l a b^{-}}$ axis the solid angle $\Delta \Omega$ has been chosen so that the detector acceptance is $\Theta_{l a b} \pm 10^{\circ}$. Also drawn are the most important contributions to the total uncertainty, being mainly counting statistics and accelerator induced helicity correlated effects at low values of $\Theta_{l a b}$. At larger angles, the form factor contributions are predominant. The minimum of the total error at $\Theta_{l a b}=20^{\circ}$ corresponds to the projected precision of $0.15 \%$ in the determination of $\sin ^{2}\left(\theta_{W}\right)$.

\begin{tabular}{ll}
\hline$E_{\text {beam }}$ & $200 \mathrm{MeV}$ \\
$I_{\text {beam }}$ & $150 \mu \mathrm{A}$ \\
$P$ & $(85 \pm 0.5) \%$ \\
Target & $60 \mathrm{~cm}$ liquid-hydrogen \\
$\Delta t$ & $10000 \mathrm{~h}$ \\
$\theta_{\text {lab }}$ & $20^{\circ} \pm 10^{\circ}$ \\
$\Delta \phi_{\text {lab }}$ & $2 \pi$ \\
$Q^{2}$ & $0.0029 \mathrm{GeV}^{2}$ \\
$A_{P V}$ & $-20.25 \mathrm{ppb}$ \\
$\Delta A_{P V}$ & $0.34 \mathrm{ppb}(0.25 \mathrm{ppb}$ stat., $0.19 \mathrm{ppb}$ syst., $0.17 \%$ rel. $)$ \\
$\Delta \sin ^{2}\left(\theta_{W}\right)_{\text {tot }}$ & $3.6 \cdot 10^{-4}\left(2.8 \cdot 10^{-4}\right.$ stat., $0.15 \%$ rel. $)$ \\
$\Delta Q_{W}(p)$ & $1.44 \cdot 10^{-3}(1.9 \%$ rel. $)$ \\
\hline
\end{tabular}

Table 1: Input parameters for and results of the calculations shown in figure 6.

\section{Detector concept studies}

The need for high statistics results in the need for a high luminosity $L \approx 10^{39} \cdot \mathrm{s}^{-1} \cdot \mathrm{cm}^{-2}$. This means that at full beam current an integrating measurement has to be performed. Therefore a good separation from background - which in our case is mainly composed of electrons stemming from Møller scattering and Bremsstrahlung-photons - is required.

The P2 collaboration is investigating on the realizability of a solenoid spectrometer. 


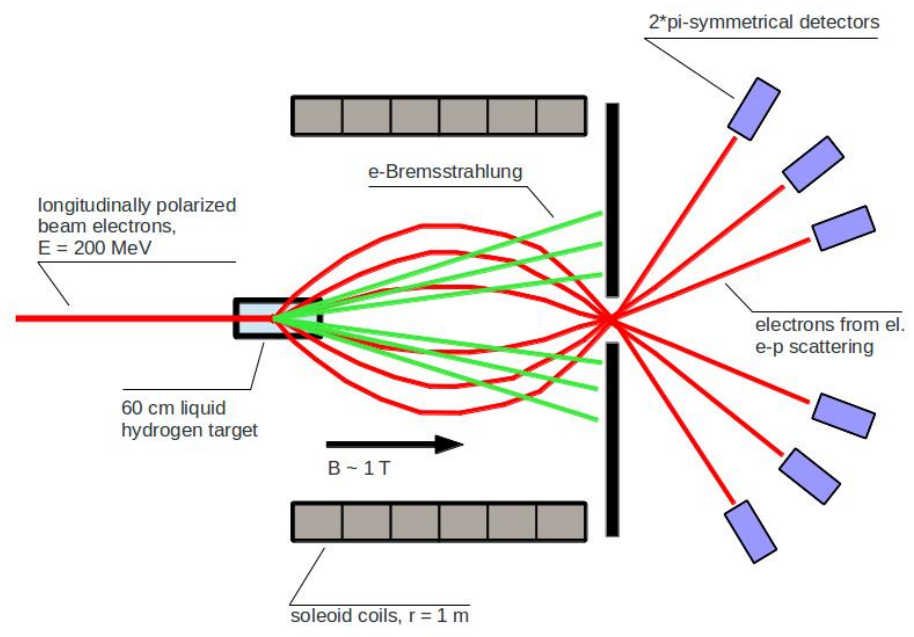

Figure 7: Sketch of Project P2's detector concept: Electrons (red trajectories), scattered elastically off protons in a liquid hydrogen target, pass through the magnetic field of a solenoid and are registered in a detector covering the full azimuth. One has to apply shields to prevent photons (green trajectories) produced inside the target cell from hitting the detector. This concept has two advantages over a toroid-spectrometer. The first is that there are no coils in the electrons' way so that nearly the full azimuth can be used for collecting statistics. Secondly, a solenoid spectrometer is the more compact setup.

Figure 7 illustrates the idea. The feasibility study is done using ROOT and Geant 4 . We are interested in performing realistic tracking simulations of both signal and background particles in the solenoid's magnetic field. Therefore a realistic model of a solenoid's magnetic field is required. A program for numeric calculation of magnetic fields has been developed, figure 8 shows results for a ZEUS-like configuration of superconducting coils. Fieldmaps like these have been included in the Geant4 simulations we are going to present in the following.
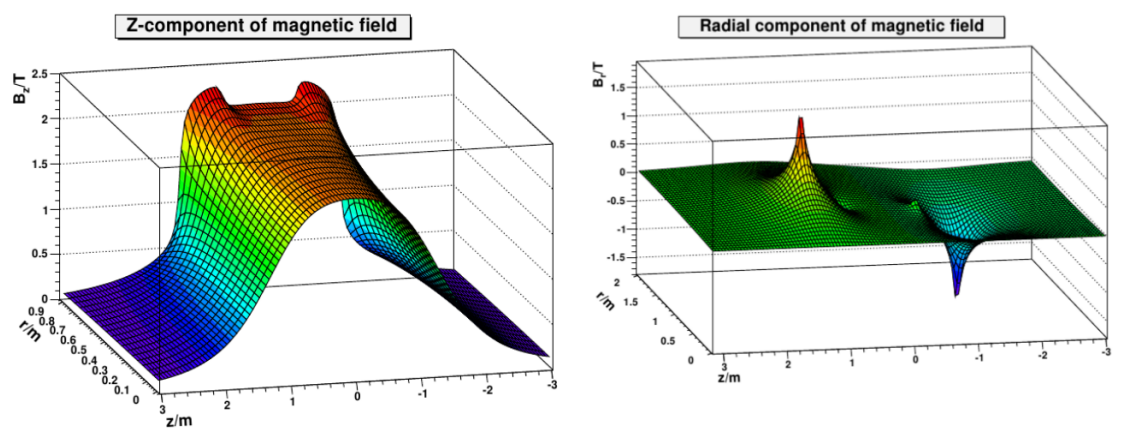

Figure 8: A ZEUS-like solenoid's magnetic field in cylindrical coordinates: The picture on the left shows the component parallel to the beam axis, the picture on the right the radial component of the field. The azimuthal component turns out to be negligibly small. The calculation has been done by solving the BiotSavart law in three dimensions. The solenoid is $2.4 \mathrm{~m}$ long, it's radius is $0.96 \mathrm{~m}$. The maximum field strength along the beam axis is $1.8 \mathrm{~T}$. 


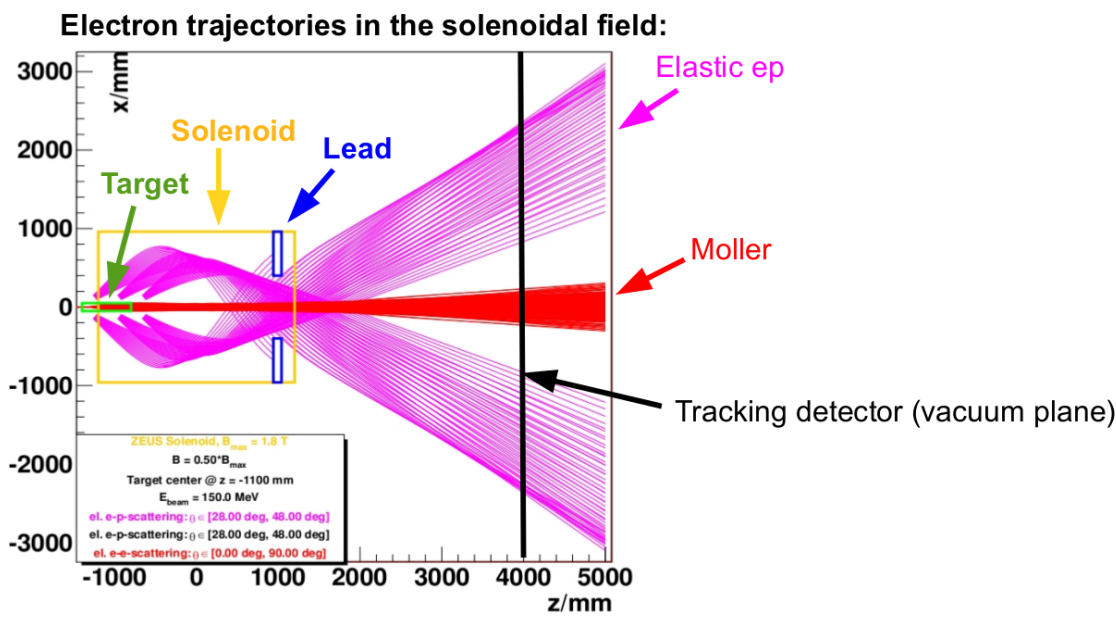

Figure 9: Tracking simulation in the magnetic field of a ZEUS-like solenoid: Primary events for a fixed set of scattering angles have been generated at three points along the beam axis (beginning, middle and end of the target volume) assuming a linear energy loss of the beam electrons inside the target cell. The sampling of secondary particles by Geant 4 has been turned off. Since the electrons stemming from Møller scattering have much less energy than those scattered elastically off protons under the same angle, these background electrons' trajectories stay very close to the beam axis even in the solenoid's stray field. The black line at $\mathrm{z}$ $=4000 \mathrm{~mm}$ marks a possible detector position.

The first thing we are interested in is the tracking of charged particles in the magnetic field. Figure 9 shows a projection of electron trajectories in the solenoid's magnetic field. Even in the solenoid's stray field the electrons stemming from Møller and el. e-p scattering remain separated, which would allow to place a ring detector covering the full azimuth behind the solenoid.

Figure 9 shows results of a very idealized case, since - apart from the generation of primary events assuming a linear energy loss inside the target cell and the tracking algorithms - no physics processes were enabled in the simulation. Yet these simulations give an overview of the trajectories' shapes and allow to determine the positions of shields to cover the line of sight between the target volume and the virtual detector. This is necessary to prevent gammas produced in the target by the electron beam from hitting the detector.

The configuration (target position, magnetic field strength, placement of the shielding) determined with this naive tracking simulation may then be used in a more realistic Geant 4 simulation. Figure 10 shows a rate prediction generated with a more sophisticated simulation for the tracking detector shown in figure 9, using the same setup as presented in figure 9. In this simulation, tree level event generators for elastic electron-proton and Møller scattering have been applied for primary event generation. The primary vertices were uniformly distributed along the beam axis inside the liquid-hydrogen volume, assuming a linear loss of energy of the beam electrons passing through the target. Physics processes like Bremsstrahlung, ionization, multiple scattering, pair production and annihilation were included in the simulation. The signal electrons from el. e-p scattering (magenta curve) are well separated from the Møller electrons (light red curve). As already predicted by the tracking simulation, the Møller electrons' trajectories stay close to the beam axis. The green curves mark the contributions from photons with energies larger than $10 \mathrm{MeV}$. The rapid decrease 


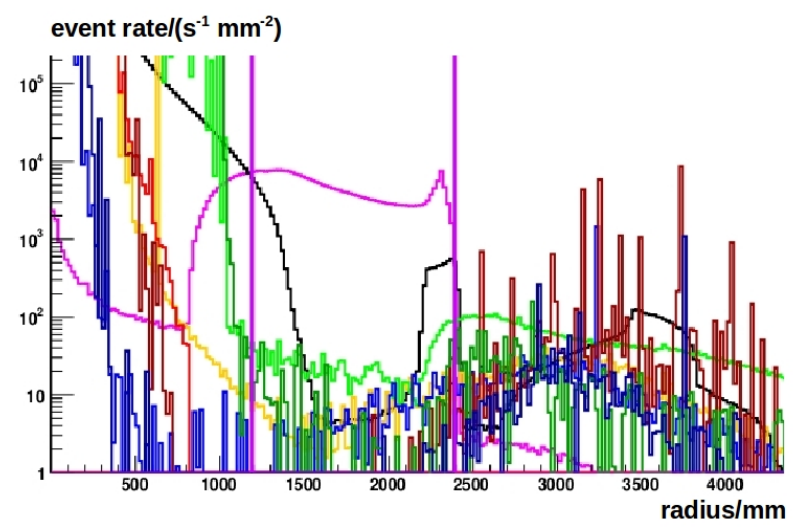

Figure 10: Rate prediction for the tracking detector shown in figure 9: Drawn is the rate per $\mathrm{cm}^{2}$ vs. the distance from the beam axis. The magenta distribution belongs to the electrons from elastic e-p scattering with a scattering angle between $28^{\circ}$ and $48^{\circ}$. These are the electrons we need to separate from the background. For electrons from elastic e-p scattering with scattering angles outside of $\left[28^{\circ}, 48^{\circ}\right]$, the distribution is shown in black. The yellow distribution represents protons from elastic e-p scattering. The red curves belong to electrons stemming from Møller scattering, photons are depicted in green and positrons are shown in blue. The two vertical lines in purple at $r=1200 \mathrm{~mm}$ and $r=2400 \mathrm{~mm}$ mark possible boundaries for a detector. Further discussion in the text.

of their rate around $\mathrm{r}=1000 \mathrm{~mm}$ is due to the shielding shown in figure 9. The violet lines mark a possible detector position. Since the rates predicted by the simulation are very high $(0.44 \mathrm{THz}$ total for elastic e-p scattering), the P2 collaboration is studying the usability of Cherenkov counters as modules for a ring detector. Geant 4 simulations of a single module have been performed and several prototypes have been successfully tested at the MAMI accelerator facility. For details, see the contribution by K. Gerz to these proceedings.

\section{Outlook}

The P2 collaboration is planning to make a decision on the principal experimental setup in the middle of 2014. In order to provide a solid basis for this decision, the Geant 4 simulations have been further developed to make the rate predictions more realistic: We are going to include radiative corrections in the event generators. A simulation of the beam-target interaction has been developed in order to produce realistic vertex distributions for the generation of primary events. Also the expected detector response will be included in the calculation of the signal distributions shown in figure 10 .

\section{References}

[1] K. S. Kumar, Int. J. Mod. Phys. E19 (2010) 927-937

[2] J. Erler, A. Kurylov and M. J. Ramsey-Musolf, Phys. Rev. D68, 016006 (2003)

[3] M. Gorchtein, C. J. Horowitz and M. J. Ramsey-Musolf, Phys. Rev. C84, 015502 (2011) 